\title{
CAMBIO EVOLUTIVO, CONTEXTOS E INTERVENCIÓN PSICOEDUCATIVA EN LA VEJEZ
}

\author{
Carmen Triadó Tur ${ }^{1}$ \\ Departamento de Psicología Evolutiva y de la Educación \\ Facultad de Psicología, Universidad de Barcelona
}

\begin{abstract}
RESUMEN. En este artículo se aborda el estudio de la vejez desde la Psicología Evolutiva. Contempla las aportaciones del enfoque del Ciclo Vital y la influencia de los contextos de desarrollo en el cambio evolutivo en lo que se refiere a la familia, jubilación y contexto psicosocial y comunitario. Finalmente se plantean algunas reflexiones sobre la intervención psicoeducativa, se parte de las aportaciones de los investigadores respecto a las capacidades cognitivas de las personas mayores enfatizando la postura optimista y positiva que posibilita la capacidad de aprendizaje y formación en edades avanzadas.

ABSTRACT. This article studies the elderly from the evolutive perspective. It takes into account the life-span approach and the influence of developmental contexts in the evolutive change. The evolutive change undergone refers to the family, to the retirement and to the psychosocial and community context.

Finally some reflexions are made regarding the psychoeducative intervention, starting from researches, take an optimistic perspective that emphasizes the relevant role of learning and education.
\end{abstract}

\section{Introducción}

En este artículo presentamos algunas reflexiones sobre el estudio de la vejez desde la Psicología y la Educación.

Iniciamos nuestra exposición con unos breves apuntes de los inicios de la investigación científica sobre la vejez en cuanto a sus implicaciones psicológicas, para a continuación plantear nuestra reflexión desde la Psicología Evolutiva. Para lo cual partimos de la psicología del Ciclo Vital, tomando la definición de Baltes, Reese y Nesselroade (1981), "la psicología evolutiva se ocupa de los cambios de comportamiento que presentan las personas en el transcurso del ciclo vital, así como de las diferencias y semejanzas interpersonales respecto a estos cambios. Su finalidad no consiste sólo en describir estas variaciones y diferencias interindividuales, sino también en explicar cómo aparecen y en describir vías para modificarlas óptimamente, se ocupa también de los cambios verificados dentro de las ecologías bioculturales y entre las mismas, así como de las relaciones existentes entre estos cambios y los que

1. Carmen Triadó Tur. Departament de Psicología Evolutiva i de l'Educació. Facultat de Psicología. Universitat de Barcelona. Passeig de la Vall d'Hebron, 171. 08035 Barcelona. e.mail: mtriado@psi.ub.es 
se producen dentro y entre los individuos" (pag.23).Esta perspectiva como señala Belsky (1996) es una prescripción para tener una actitud abierta: el pluralismo (una diversidad de puntos de vista) es la mejor norma que cabe seguir en la descripción y explicación del funcionamiento psicológico en el último periodo de la vida.

Así pues, vamos a desarrollar los principales aspectos de la perspectiva del ciclo vital para a continuación exponer los contextos en los que se dan los cambios evolutivos en la vejez y su relación con la intervención psicoeducativa.

\section{El estudio psicológico de la vejez}

Para James Birren (1961) en el estudio de la vejez desde la investigación de los procesos de envejecimiento psicológico, se distinguen tres etapas:

La primera la considera como período inicial de la investigación y empieza en 1835 cuando Quetelet (1796-1874) publica la obra "El hombre y el desarrollo de sus facultades". Lehr (1988) considera que la importancia de Quetelet para la investigación del envejecimiento estriba, por una parte, en que se opuso a la inaceptable generalización de las comprobaciones aisladas y por otra combatió cualquier procedimiento meramente casuístico, destacando de modo convincente la relación entre las influencias biológicas y sociales, en el proceso de envejecimiento.

La influencia de Quetelet se notó en Francis Galton (1832-1911), que aportó a la psicología del envejecimiento investigaciones específicas, correlacionó las modificaciones físicas que se experimentan con la edad con los cambios en la psicomotricidad, percepción y procesos mentales, y repitió las investigaciones en los mismos sujetos a lo largo de los años.

La segunda etapa es considerada por Birren como el comienzo de la investigación sistemática y se inicia en 1918 para continuar hasta 1940. En 1922 el psicólogo norteamericano Stanley Hall, publica "Senescence, the last half of life", es el primer estudio realizado desde el punto de vista de la psicología. Hall se negó a aceptar un modelo deficitario de la vejez y destacó que las diferencias individuales en la vejez son significativamente mayores que las que aparecen en otras etapas de la vida, al tiempo que estaba convencido de la identidad o especificidad de una psicología del proceso de envejecimiento, ya que la vejez tiene sus propios modos de sentir, pensar o querer.

En 1928 Miles fundó en la Universidad de Stanford, de California, el primer Instituto destinado especialmente al estudio de los problemas del envejecimiento. A partir de 1930 se van sucediendo en Norteamerica la fundación de diversas asociaciones con finalidad gerontológica.

En el ámbito europeo, destacamos a la psicóloga alemana Carlota Buhler que en 1933 publica "El curso de la vida humana como problema psicológico" en la que se ocupa de estudiar el ciclo completo de la vida, para obtener una visión de conjunto comprensiva desde la infancia hasta la vejez. En España Santiago Ramón y Cajal (1852-1934) publica dos años antes de su muerte el ensayo "El mundo visto a los 80 años", producto de su autoobservación . El libro contiene descripciones sobre la vejez y sus bases psicológicas y sociales.

Por último Birren, considera una tercera etapa que llama, expansión de las investigaciones sobre el envejecimiento. Esta fase la inicia el norteamericano Cowdry (1939) con su libro "Problems of Aging" (Problemas del envejecimiento) concebido 
desde un punto de vista médico, aunque posteriormente amplia el concepto a los aspectos sociales, psicológicos y psiquiatricos.

A partir de este momento el estudio de la vejez se aborda de forma más sistemática. En 1945 se crea la Gerontologic Society. El 1948 la Sociedad Española de Geriatría y Gerontología. Se publican revistas en las que se reflejan las investigaciones realizadas, cabe destacar el Journal of Gerontology en 1946.

Finalmente como señala Rodríguez Domínguez (1989) la creación en 1977 de la "Asamblea Mundial del Envejecimiento" bajo el patrocinio de la Asamblea General de las Naciones Unidas, propicia el mayor impulso institucional contemporáneo a la investigación pluridisciplinar sobre la vejez, puesto que las recientes celebraciones de la Conferencia Mundial sobre Envejecimiento y de los Simposiums Internacionales de gerontología vienen suscitando la comparecencia en tales convocatorias no sólo de especialistas a título individual, sino también la representación oficial u oficiosa de las instituciones nacionales interesadas en dar respuesta satisfactoria al grave problema actual del sector social de las personas ancianas en los ámbitos diversos del bienestar físico, psíquico, social y económico.

En la sociedad contemporánea la vejez llega a tener representación social y deja de ser un problema individual o familiar para cobrar significado público. Los estudios demográficos recientes nos indican que la estructura de la población está cambiando aceleradamente, ello comporta implicaciones individuales, sociales, académicas y científicas. Como señalan Vega y Bueno (1995), al prolongarse el curso vital de las personas, éstas planifican su futuro y tienen expectativas diferentes. El envejecimiento de la población comporta también cambios a nivel social en los que se destaca la reorganización social de sus generaciones. Además la psicología evolutiva y de la educación tiene que abordar un nuevo fenómeno que por su complejidad supone un desafío. Ello conlleva generar un cuerpo de conocimientos científicos sobre capacidades sensoriales, memoria, funcionamiento cognitivo y optimización de capacidades e influencia de los contextos que afectan a los cambios evolutivos en la vejez.

\section{La psicología del Ciclo Vital}

El enfoque de la psicología del desarrollo a lo largo de ciclo vital surge entre los años 60-70. Esto no quiere decir que en épocas anteriores no se haya considerado el desarrollo en las edades posteriores a la infancia y adolescencia, como se ha constatado en los estudios de Stanley Hall y Carlota Bhuler. Este enfoque ha adquirido gran importancia siendo las aportaciones más relevantes las de P.B. Baltes al que podemos considerar como su portavoz más activo.

Para el enfoque del ciclo vital el desarrollo abarca la totalidad de la vida, desde el nacimiento hasta la muerte, ya que comprende todos y cada uno de los procesos de cambio que se dan a lo largo de la vida. Estos procesos de cambio no siguen necesariamente patrones fijos y predeterminados (progreso en la infancia y declive en la vejez), sino que pueden diferir entre ellos al menos en las siguientes dimensiones:

- Dirección: hay cambios que implican crecimiento en términos psicológicos, mientras que otros implican declive.

- Temporalidad: el cambio puede producirse en cualquier punto del ciclo vital, tener una duración variable y acabar también en cualquier punto de la vida. 
Todos los cambios tanto los cortos como los de larga duración, los positivos y los negativos, pueden considerarse dentro del concepto amplio de desarrollo. Al introducir el término "desarrollo" los psicológos del ciclo vital se ven obligados a reformularlo, y es así como Baltes (1983) cree que, existe la necesidad de una concepción del desarrollo que incluya los tradicionales enfoques evolutivos centrados en el crecimiento como una clase importante, pero especial, de fenómenos evolutivos. Es necesaria una taxonomía de modelos de cambio evolutivo más comprensiva que nos permita transcender las limitaciones restrictivas establecidas por los biológos y asumidas por muchos especialistas en el desarrollo infantil.

La reformulación del propio concepto de desarrollo se basa en que los cambios que tienen lugar a lo largo del ciclo vital pueden ser de naturaleza bastante diversa. Mientras que el desarrollo intelectual infantil puede evidenciar rasgos de secuencialidad, unidireccionalidad, irreversibilidad y universalidad, los procesos de desarrollo que tienen lugar durante la madurez y la senectud pueden evidenciar rasgos de multidimensionalidad y multidireccionalidad, reflejar grandes diferencias interindividuales y obedecer a una gran plasticidad contextual. Por tanto, el concepto clásico de "crecimiento" tomado de la biología, mientras puede resultar útil para describir algunos procesos de desarrollo, especialmente durante las primeras etapas posee algunos rasgos inapropiados o excesivamente restrictivos para el estudio del cambio ontogenético en la estructura del ciclo vital (Baltes, 1983), lo cual requiere una expansión o modificación del concepto tradicional y monolítico del desarrollo.

Otra característica de la perspectiva del ciclo vital es el énfasis en la influencia que podrían tener sobre el desarrollo de la persona no únicamente factores vinculados a la edad, sino también aquellos relacionados con el entorno histórico social en el que se encuentra inmersa la persona o incluso con experiencias vitales particulares. Se distinguen tres factores de influencia en el desarrollo:

- Influencias normativas relacionadas con la edad: hacen referencia a factores biológicos o sociales que se relacionan estrechamente con la edad cronológica. Un ejemplo de estas influencias lo constituye la maduración biológica y también la socialización, cuando se considera bajo la perspectiva de la aparición de roles o competencias normativas relacionadas con la edad.

- Influencias normativas relacionadas con la historia: se refiere a los efectos del cambio biosocial que varian en función del tiempo histórico tal y como se evidencia en los efectos generacionales. No se puede considerar de la misma manera la vejez de hace cien años a la vejez actual, por la gran cantidad de cambios y sucesos sociales e historicoculturales que han tenido lugar. Este factor es responsable del parecido de las personas de una misma generación.

- Influencias no normativas: se refieren a factores biológicos o sociales que afectan a personas concretas, no a la generalidad, en un momento preciso de su vida, sin seguir patrones ni secuencias fijas, por ejemplo, acontecimientos que pueden afectar la vida laboral (jubilación anticipada), la vida familiar (divorcio, muerte de un hijo), o la salud (accidente grave). Como representantes de las experiencias vitales únicas, estos factores son responsables de gran parte de las diferencias interindividuales, sobretodo en personas de la misma generación.

Los teóricos de esta perspectiva admiten que la fuerza de cada una de las categorías de determinantes no es la misma en todos los puntos del ciclo vital, sino 
que cambia en función de la edad. Baltes (1983) afirma que durante la infancia los factores más relevantes son los relacionados con la edad, que son precisamente los que remarcan la regularidad y la homogeneidad de los cambios. Mas allá de la adolescencia, la regularidad de los factores biosociales se perdería y se entraría en etapas más abiertas y variables, en las cuales las influencias normativas relacionadas con la historia y las no normativas determinarían la gran cantidad de cambios evolutivos que se producen. Si aceptamos esta relación, los factores sociales e históricos y los acontecimientos vitales propios de cada persona son los elementos básicos para entender el desarrollo en la edad adulta y la vejez. De esta manera la vejez no se contemplaría como una etapa homogenea marcada por el declive, sino como una etapa en la cual seguramente se dan más diferencias individuales, ya que se manifestarían las improntas de toda una vida de experiencias vitales particulares (Villar,1998).

Finalmente es necesario decir que, en los últimos años, quizás a causa de la amplia aceptación de los principios de la perspectiva del ciclo vital en la psicología evolutiva en general y en la psicología del envejecimiento en particular, ha emergido un nuevo enfoque, el llamado envejecimiento satisfactorio o envejecimiento con éxito. Como señala, Fernández Ballesteros (1998), se ha focalizado y comenzado la investigación sistemática de una serie de aspectos positivos del envejecimiento como son el potencial de aprendizaje, la sabiduría, la satisfacción con la vida o la felicidad en el área psicológica.

Muchos de los representantes de la psicología del ciclo vital han abordado el estudio de los condicionantes que permiten envejecer de manera óptima (Baltes y Baltes,1990; Baltes, 1993). Desde este punto de vista, las personas que llegan a disfrutar de un envejecimiento con éxito, se implicarían en una serie de estrategias que describen el proceso general de adaptación a lo largo de todo el ciclo vital, pero que resultan especialmente relevantes cuando, a causa del proceso de envejecimiento, las pérdidas y las amenazas de pérdidas aumentan. Las tres estrategias más nombradas son la selección, la optimización y la compensación (Baltes y Baltes, 1990; Baltes, 1997; Schultz y Heckhausen, 1996).

- Selección de los ámbitos vitales prioritarios en función de las demandas ambientales, las habilidades previas o el nivel de satisfacción que se obtiene de ellas, dejando, en cierta medida de lado las otras. Se trata de centrarse en uno o pocos dominios para continuar funcionando con ellos con el mismo nivel de cualidad o incluso mejor que en años anteriores, a costa de sacrificar el número global de dominios en los que nos encontramos generalmente implicados.

- Optimización de la actuación en los dominios escogidos, de las trayectorias vitales que se siguen, gracias a una capacidad de aprendizaje y enriquecimiento (plasticidad) que no se pierde con los años.

- Compensación de las posibles pérdidas en ciertas capacidades comportamentales a través de otras capacidades que se conservan más intactas o que hayan aumentado con la edad o mediante ayudas de carácter tecnológico.

Esta nueva perspectiva, como señala Fernández Ballesteros (2000) puede ser considerada una nueva visión de la vejez y el envejecimiento como fenómeno individual y social, portador de una serie de aspectos positivos, tanto al nivel del individuo como de la población, que han comenzado a ser estudiados y tenidos en cuenta por los investigadores de la vejez y el envejecimiento. 


\section{Contextos psicoeducativos}

El ser humano cambia y se desarrolla en diferentes contextos que influyen sobre las personas y sus relaciones. Quien ha descrito y examinado este tipo de influencias ha sido Bronfenbrenner (1986) proporcionando un modelo de desarrollo humano que es referencia obligada en la psicología evolutiva actual. Para Bronfenbrenner existen varios tipos de sistemas que guardan relación entre sí: microsistema, mesosistema, exosistema y macrosistema.

- El microsistema, comprende el conjunto de relaciones más próximas, es decir, las relaciones entre la persona y su ambiente inmediato, en todas las edades de la vida la familia es el ambiente más inmediato, así en la vejez la vida en pareja, los hijos y los nietos, son subsistemas que guardan una relación jerárquica entre sí, si alguno de ellos falla repercute en el bienestar psicológico de las personas, en este caso de los mayores, otro ambiente inmediato es el ámbito laboral.

- El mesosistema, se refiere al conjunto de relaciones que se establecen entre dos o más microsistemas en los que participa la persona, por ejemplo, las relaciones entre familia y trabajo, lo que ocurre en la familia puede repercutir en la actividad laboral y así mismo los problemas o satisfacción en el trabajo repercuten en las relaciones y actitudes familiares.

- El exosistema, está formado por las estructuras sociales formales e informales que influyen o delimitan lo que ocurre en los ambientes próximos, por ejemplo, las amistades, las relaciones vecinales, los servicios sociales, etc.

- El macrosistema, es el más distanciado del individuo e incluye los valores sociales e ideológicos de una sociedad, son las influencias histórico culturales que caracterizan a las distintas generaciones.

Como señalan, Rodrigo y Palacios (1998), cada uno de estos sistemas tienen la peculiaridad de cambiar a lo largo del tiempo, tanto si se trata del tiempo ontológico (historia del individuo) como si nos referimos al tiempo histórico (historia de la comunidad).

En este sentido, es necesario remarcar que cada época histórica y los diferentes grupos sociales organizan la socialización de forma diferente, la relación entre individuo y cultura está fuertemente condicionada por las características de cada momento y contexto, así pues desde la perspectiva a la que nos venimos refiriendo las relaciones que modelan el desarrollo son interacciones contextualmente condicionadas tanto desde el punto de vista del individuo como de la historia.

A continuación vamos a desarrollar cómo influyen los distintos contextos en los cambios evolutivos que se dan en la vejez.

\subsection{Contexto psicoeducativo familiar}

Las personas mayores de 65 años y especialmente de 70 , se encuentran que han de hacer frente a una serie de cambios que ha experimentado la estructura familiar y unos más otros menos intentan adaptarse a estos cambios. Una gran mayoría de estas personas vivieron en la infancia con una familia donde estaban presentes padre y madre así como compartieron su entorno familiar con algún abuelo/a. La mayoría se han casado y han tenido hijos y muchos de ellos han podido comprobar que sus hijos no seguían el mismo tipo de relación familiar, ya sea por separación o divorcio o por soltería con vida independiente o por relaciones homosexuales, lo cual implica una serie de cambios en sus cogniciones y/o creencias. 
Durante muchos años y especialmente en la vejez la familia es el microsistema más importante. En la vejez, la mayoría de las personas continúan casadas, especialmente los hombres, ya que hay más mujeres viudas por la mayor esperanza de vida de éstas y por el hecho de que los hombres viudos suelen volver a contraer matrimonio. Respecto a la vida marital, muchas parejas mayores coinciden en que su matrimonio se ha ido consolidando con los años. Huyck y Hoyer (1982) recogieron un número considerable de conclusiones sobre la satisfacción marital en los mayores que resumimos en las siguientes:

- Parece ser que las personas mayores casadas presentan mejor salud y experimentan menos la soledad. La pareja constituye un buen sistema de mutua asistencia.

- Según las estadísticas valoran mejor el matrimonio los hombres que las mujeres.

- La relación entre matrimonios de edad va desde el mutuo entendimiento hasta la relación deteriorada que puede acabar en divorcio o separación.

Acumular experiencias compartidas a lo largo de la vida hace que los matrimonios sean más compatibles, es decir, todos los factores contextuales que han compartido, como son, educar a los hijos, vivir en el mismo núcleo social, hacer frente a las mismas necesidades económicas, compartir las mismas creencias, suelen cambiar a ambos cónyuges de manera similar. (Caspi \& col., 1992).

La solidaridad entre las parejas de edad avanzada se pone de manifiesto cuando aparecen problemas de salud. Los cónyuges de estas edades aceptan sus respectivas deficiencias y atienden a las necesidades físicas del otro, con fuerte vinculación emocional. A estas edades es frecuente que uno de los miembros de la pareja deba afrontar la muerte del otro y por tanto enfrentarse a la pérdida de un figura importante de apego. Como señala López (1998), si las relaciones entre los esposos son adecuadas, las relaciones de apego, amistad, intimidad sexual y de cuidado mutuo pueden seguir profundizándose y verse beneficiadas por la seguridad de los numerosos años de convivencia y las experiencias vividas juntos, y si además las relaciones con los hijos son satisfactorias y se acepta su autonomía, sin grandes conflictos, el proyecto de vida en común y, más en concreto, el proyecto de paternidad y maternidad, se verán colmados.

El contexto familiar en la vejez experimenta cambios que empiezan en la mediana edad. Uno de los cambios más importantes es la independencia de los hijos, ya sea por formar una nueva familia o para vivir una vida propia e independiente. Este cambio comporta también la incorporación de nuevos miembros como son yernos, nueras y nietos, y así se establecen relaciones intergeneracionales que en ocasiones pueden llegar hasta cinco generaciones: es lo que se conoce como familia vertical. Como señalan Berger y Thomson (2000), si bien las relaciones entre generaciones constituyen un valor positivo, también es probable que comporten tensiones y conflictos. Casi todas las personas mayores con hijos y con nietos les dedican tiempo y atención. Pocos adultos dejan de ejercer de padres por el hecho de que sus hijos hayan crecido, sean independientes o incluso se hayan casado o que además sean padres.

En el contexto familiar se establecen por un lado las relaciones padres-hijos y por otro las de los abuelos con sus nietos. Los datos de las investigaciones muestran que en la sociedad actual se mantienen las relaciones familiares y los sistemas de apoyo de los hijos para con sus padres mayores. Se constata también que las relaciones se establecen en mayor medida por la línea materna, siendo además las hijas las que 
mayor contacto y apoyo social tienen con sus padres y especialmente la madre cuyas relaciones suelen ser muy íntimas y a la vez vulnerables a tensiones. El contacto familiar depende de la proximidad geográfica más que de ningún otro factor. Cuando se vive cerca o en la misma población los contactos con los padres son frecuentes pero se mantienen con gran frecuencia los contactos telefónicos y los padres mayores suelen decir que la relación ha mejorado y tienen frecuentes contactos con sus hijos especialmente las hijas. Los contactos telefónicos se dan más con los hijos en los que hay distancia geográfica y también son más frecuentes con las hijas (Triadó, 2000).

Para Mangen y col. (1988) las relaciones familiares pueden estudiarse en función de la ayuda mutua, el contacto frecuente y el afecto compartido. La ayuda surge tanto por necesidad como por deseo y capacidad de prestarla y esta ayuda tanto puede ir de padres a hijos como a la inversa. Los padres mayores representan en muchos casos una fuente de ayuda principal. En cuanto a los hijos son también fuente de ayuda para los padres mayores y se comprometen económicamente, física y emocionalmente con ellos.

El afecto compartido suele estar muy influido por el historial anterior de amor y respeto mutuo de la familia. Esto significa que una familia puede mostrar un elevado grado de los dos primeros signos de unidad, ayuda y contacto, sin que exista de forma necesaria gran calidez y afecto entre sus miembros.

Las muestras de solidaridad son más frecuentes en determinados casos. Sobretodo cuando las personas mayores sufren discapacidades físicas o mentales, pierden autonomía e independencia, por lo que necesitan atención y ayuda y por tanto la importancia del apoyo familiar aumenta en función del grado de dependencia de la persona mayor. La asignación del rol de cuidador depende de la posición en la estructura familiar. En primer lugar es asumido por el cónyuge, después por los hijos, nietos, hermanos, sobrinos... En el caso de los hijos, son con mayor frecuencia las mujeres quienes asumen el rol de cuidadoras.

Según el estudio realizado por el INSERSO (1995), las personas mayores son atendidas, principalmente, por miembros de la familia: hijas (43,5\%); cónyuge $(21,7 \%)$; nueras $(7,5 \%)$; hijos $(5,8 \%)$; yernos $(0,2 \%)$ y otros parientes como hermanos, sobrinos, primos, nietos, etc. $(7,8 \%)$. Los motivos por los cuales cuidan a sus mayores hacen referencia a sentimientos de afecto, amor y proximidad; así como de responsabilidad y deberes morales y éticos. Las personas mayores tienen sus preferencias sobre quién quieren que les cuiden, Triadó (1997) en entrevistas realizadas a personas mayores de 65 a 80 años, observó que más del 50\% prefieren que sean los hijos. El motivo fundamental es que "como la familia no hay nada".

En la vejez, es frecuente que la persona se deteriore o que sea dependiente. A menudo puede ofrecer poco en reciprocidad y es frecuente que los cuidados que necesite requieran esfuerzo y sacrificio. Por ello, como señala López (1998) es decisivo que pueda contar con personas, figuras de apego, que le sean material y sobre todo, emocionalmente incondicionales y los cuidados emocionales que una persona necesita en las situaciones límites de la vida, sólo los pueden prestar satisfactoriamente las figuras de apego. Las personas ancianas pueden acabar eligiendo a sus hijos como figuras de apego. Parece razonable pensar que los hijos más comprometidos y eficaces en el cuidado de los padres sean convertidos por estos en figuras de apego, pero también parece esperable que padres más débiles y vulnerables a la enfermedad estén más inclinados a establecer relaciones de apego con los hijos. 
Las relaciones con los nietos son fuente de satisfacción. Los datos del estudio de Triadó, Villar (2000), muestran que el vínculo afectivo de los abuelos para con sus nietos es muy elevado, aunque las actividades compartidas no son muchas. La corriente de intercambio va de los abuelos a los nietos y destaca la función del abuelo como portador de la memoria familiar. Las abuelas enfatizan más la vinculación emocional con sus nietos y se muestran más proclives que los abuelos a demandarles ayuda.

El contexto familiar es un microsistema donde se desarrollan diferentes sucesos vitales en la vejez, y donde la mayoría de personas ancianas se sienten protegidas, seguras y emocionalmente estables. El apoyo familiar en la vejez es una fuente importante de bienestar psicológico.

\subsection{Contexto psicoeducativo en el ámbito laboral}

El contexto laboral forma parte del microsistema, desde un punto de vista social. En este contexto, la vejez coincide con el fin de la actividad laboral, por tanto, el exosistema se ve afectado por la interrupción de un tipo de relaciones interpersonales o de amistad que se desarrollan en los ámbitos laborales.

En general en los países industrializados, alrededor de los 65 años las personas finalizan su actividad laboral y adquieren el estatus de jubiladas, y gracias a la mayor esperanza de vida, las personas viven muchos años desde que se jubilan, y en los casos de jubilación anticipada estos años se incrementan.

La jubilación cambia la vida de las personas tanto a nivel personal como social. Las actitudes ante la jubilación pueden ser muy diversas; lo cierto es que las personas jubiladas deben adaptarse a una nueva situación y para algunas puede ser difícil. Según el tipo de trabajo que se ha realizado será más o menos costosa la adaptación. Achley (1989) considera que cuanto más cualificado es el trabajo y más identificadas están las personas con su actividad laboral, más difícil es aceptar la nueva situación; si el trabajo es poco cualificado las personas esperan la jubilación con satisfacción, aunque ésta depende también de otros factores; así podemos observar que las personas con mayor satisfacción son las que tienen buena salud y condiciones físicas que les permiten disfrutar mejor del tiempo libre; también están satisfechas las personas que tienen una pensión y recursos económicos suficientes por lo que no perciben pérdida asociada al nuevo estatus. También viven mejor la jubilación aquellas personas que saben organizar su tiempo libre con actividades tanto recrea-tivas como sociales.

Las posibilidades de adaptación a la jubilación están delimitadas por los factores biológicos, psicológicos, culturales y sociales que convergen en una sola persona.

Muchas veces no resulta sencillo asumir el cambio que significa en la vida de las personas dejar de trabajar, por lo que tiene especial importancia la puesta en marcha de programas de preparación a la jubilación que ayuden a plantear nuevos roles y nuevas actividades. Estos programas pueden constituir un instrumento de prevención en la medida que permiten la anticipación de nuevas situaciones y su comprensión y elaboración. La promoción de nuevas modalidades y posibilidades de participación en esta etapa y el despliegue de una posición socialmente activa-opuesta a los estereotipos habituales- son algunas de las cuestiones a tratar en los programas de preparación para la jubilación en una orientación de desarrollo comunitario (Triadó, Martínez, Villar, 2000). 
Ambos microsistemas, familiar y de trabajo, se influyen mutuamente. Un estatus derivado del trabajo es la jubilación. La adaptación a la jubilación y las alternativas de ocupación del tiempo influyen en las relaciones familiares tanto positiva como negativamente. La jubilación de uno u otro de los miembros de la pareja tiene repercusiones en el otro y por tanto es necesario considerar los cambios y adaptaciones que las personas deben realizar en bien de su bienestar psicológico. En las personas mayores en las que la estructura familiar se ha configurado de manera que el hombre trabaja y la mujer atiende los quehaceres domésticos y familiares, la jubilación del marido necesita de un proceso de adaptación de la esposa a la nueva situación. Acostumbrada a tener el marido fuera de casa el hecho de que ahora este permanentemente en el hogar crea situaciones de dependencia y malestar en muchas mujeres lo que en ocasiones es motivo de desavenencias conyugales que afectan al bienestar psicológico de la pareja. En el aspecto emocional parece ser (Berger y Thomson 2000) que las mujeres acostumbran a adaptarse mejor que los hombres a la jubilación, porque es probable que mantengan vínculos sociales con muchas personas que no están relacionadas con su trabajo.

Actualmente las personas mayores con buena salud continúan siendo productivas aunque estén jubiladas. Sus actividades no están remuneradas económicamente pero tienen valor en muchos sentidos: formación continuada, voluntariado, participación social en actividades religiosas o políticas. Por lo tanto, la jubilación da oportunidad a las personas mayores a dedicarse a actividades que antes les era difícil realizar y que llenan su tiempo satisfactoriamente.

\subsection{Contexto psicosocial y comunitario}

El contexto social de las personas que actualmente están en la etapa de la vejez difiere de otros momentos históricos y está influido por lo que los teóricos del ciclo vital Ilaman factores normativos relacionados con la historia y la cultura. No es lo mismo ser anciano/a hoy que en el siglo XIX o principios del XX. Por un lado, en nuestro país, estas personas han vivido una guerra civil con todas sus consecuencias, por otro han tenido que adaptarse a cambios ideológicos, políticos y culturales, que influyen en sus cogniciones, creencias y actitudes individuales. Así mismo en otras épocas históricas a las personas mayores de 65 años ya se las consideraba de edad muy avanzada, hoy gracias a la atención sanitaria y a los cambios culturales, a esta edad las personas son activas aunque estén jubiladas y participan socialmente tanto en su propia formación como en actividades recreativas, de ayuda o voluntariado.

Existen actualmente un número considerable de asociaciones de personas mayores que tienen como objetivo la formación (organización de cursos de todo tipo, conferencias, viajes, actividades culturales, etc.) En diversos estudios citados por Berger y Thompson (2000), se observa que una quinta parte de personas mayores colabora en algún tipo de voluntariado organizado, en general a través de iglesias, hospitales, escuelas. También se observa que muchas más personas ancianas se dedicarían al voluntariado si reconocieran su potencial de ser útiles y si, a su vez, también lo hicieran más agencias sociales, no sólo reclutando a personas ancianas, sino además realizando un esfuerzo para combinar las capacidades de cada uno con las necesidades de la comunidad.

Así pues los mundos sociales o escenarios de la vejez que deben ser tenidos en cuenta además de la familia y los amigos son : 
- Las redes informales. Relaciones o participación social en redes sociales o grupos informales (grupo de jubilados, centros de día...).

- El vecindario. Personas que se desenvuelven en el mismo ámbito sociofísico. Este grupo constituye una posibilidad para desarrollar actividades y recursos de innovación cotidiana

- Los grupos de actividad. Vinculados al desarrollo de tareas o actividades en las que la persona puede implicarse (redes de voluntariado, participación en talleres, desarrollo de actividades lúdicas o recreativas...)

- Relaciones sociales a través de medios técnicos: Por ejemplo el teléfono, los ordenadores que constituyen una posibilidad en la configuración de relaciones interpersonales. (Corraliza Rodríguez, 2000)

En la sociedad actual se abren nuevos ámbitos y caminos para que las personas mayores puedan estar integradas, establezcan relaciones interpersonales y se vean favorecidas de los avances tecnológicos.

\section{Intervención psicoeducativa en la vejez}

Cuando se trata de la educación de los mayores observamos que están presentes determinados estereotipos y prejuicios, debido a que la imagen de las personas mayores se ha vinculado a un declive de sus capacidades no solo físicas sino también psicológicas y sociales, que sostendrían una concepción de los ancianos como difícilmente educables y poco susceptibles de adquirir nuevos conocimientos y habilidades (Villar, 2000).

Referente a las capacidades mentales y cuando se trata de evaluar la inteligencia debemos remitirnos a los primeros estudios psicométricos realizados con diseños transversales, utilizando el test de WAIS (Weschler Adult Inteligence Scale), en los que se observaba un descenso de los resultados muy acusado con la edad. Los trabajos que pueden considerarse continuadores de éstos son los dirigidos por John Horn (Horn y Catell, 1966; Horn,1982). Horn afirma que la inteligencia puede dividirse en dos grandes dimensiones: inteligencia fluida e inteligencia cristalizada.

La inteligencia fluida es la capacidad de adaptarse y rendir en tareas nuevas, que no implican ningún contenido simbólico ni semántico. Representaría el potencial intelectual biológico del individuo, determinado principalmente por factores innatos y fundamentado en la eficiencia del sistema nervioso central. Para medirla son necesarias pruebas que no tengan influencias culturales, como es la parte manipulativa del WAIS.

La inteligencia cristalizada es el resultado de la exposición del individuo a una cultura. Por tanto correspondería al conjunto de conocimientos aprendidos y almacenados por el individuo a lo largo de su vida, así pues, está determinada por factores educativos que provienen de la experiencia. La medida puede hacerse con la parte verbal del WAIS.

La diferencia entre ambas es relevante desde el momento en que Horn mantiene que una y otra siguen tendencias evolutivas diferentes: mientras que la cristalizada puede aumentar con la edad, a condición que se mantenga cierta estimulación ambiental que permita la recopilación de experiencias e información nueva, la inteligencia fluida desciende claramente con la edad. Aunque Horn hace esta diferencia- 
ción su concepción defiende el déficit en general a medida que envejecemos ya que presenta una postura fuertemente biologista, fundamentada por la pérdida neuronal irreversible y porque dedica mucha más atención a la inteligencia fluida.

El cambio en una visión optimista de las capacidades intelectuales a medida que envejecemos nos lo aportan los teóricos del ciclo vital. Su enfoque se aleja de la búsqueda de funciones de desarrollo invariantes y unidireccionales (siempre de declive) para hacer hincapié en otros aspectos, tales como:

- la importancia de las diferencias individuales y de la influencia del contexto y las circunstancias históricas en los cambios que acontecen en las personas a lo largo de la vida.

- la multidimensionalidad y multidireccionalidad de los cambios asociados a la edad, que posibilita que en una misma persona convivan pérdidas con ganancias asociadas a la edad.

- la plasticidad de las capacidades intelectuales, que posibilita que una persona pueda compensar posibles pérdidas para optimizar su desarrollo. (Triadó, Martínez, Villar, 2000)

Desde esta perspectiva se hace una crítica a algunos de los aspectos de los primeros estudios sobre la inteligencia sobretodo en dos cuestiones básicas: la metodología y los instrumentos de medida. Los estudios transversales miden en un único momento temporal grupos de edades diferentes, y por tanto confunden la variable edad con la variable generación. Por esta razón las diferencias encontradas podrían ser debidas a factores históricos y contextuales más que a cambios madurativos. Por este motivo la propuesta es realizar estudios longitudinales, es decir medidas repetidas a lo largo del tiempo sobre una muestra única de sujetos, por lo que si existen diferencias podrían ser atribuidas, en principio sólo a cambios madurativos.

En cuanto a los instrumentos de medida se considera que utilizar un único índice, no es adecuado ya que no se tiene en cuenta que pueden existir inteligencias múltiples con su propia dinámica evolutiva, su historia de declive, constancia o incluso aumento.

Partiendo de estos supuestos se han realizado estudios longitudinales. Uno de los más significativos es el Seattle Longitudinal Study dirigido por R. W. Schaie a lo largo de veintiún años (Schaie, 1989, 1994), del que se extraen las siguientes conclusiones:

- Relevancia de las diferencias intra e intergeneracionales: Schaie afirma que no hay un único patrón de déficit intelectual relacionado con la edad ni común a todos los individuos ni a todas las capacidades intelectuales. Estas diferencias son suficientemente importantes para descartar un índice global o uniforme de déficit, aunque algunas dimensiones pueden mostrar déficit en la mayoría de personas.

- Relevancia del diseño en el tipo de datos que se obtienen. Al contrario de los diseños transversales, en los diseños longitudinales se estiman correctamente los cambios madurativos en la inteligencia de aquellas personas con buena salud y que viven en un ambiente óptimo, mientras que se subestiman los decrementos en las que viven en condiciones menos favorables.

- Efectos asociados a la generación: los cambios producidos antes de los sesenta años se pueden atribuir de manera casi exclusiva a efectos generacionales, mientras que los posteriores se deben a la acción conjunta de factores generacionales y madurativos. 
Como hemos visto el enfoque del ciclo vital, destaca las diferencias entre generaciones e interindividuales en la descripción y explicación de los cambios evolutivos en general y del rendimiento intelectual en particular. Detrás de las diferencias individuales y generacionales se esconden otros factores, entre los que destacamos:

La salud. Las personas con salud delicada y enfermedades crónicas suelen rendir peor en tareas en las que han de demostrar su rendimiento intelectual.

El estilo de vida y educación. Según Schaie (1994), parece claro que estilo de vida y rendimiento intelectual están relacionados. Según sus investigaciones, tener un elevado estatus social y educacional, poseer estabilidad familiar, implicación activa del individuo en el entorno y la presencia de intereses intelectuales a lo largo de la vida se relacionan significativamente con un rendimiento óptimo, ya no sólo en el área intelectual, sino también en los demás ámbitos de la experiencia a lo largo del ciclo vital.

Actitudes ante la tarea. Algunas investigaciones sugieren que en general jóvenes y viejos afrontan con diferente actitud tareas como las que se presentan en las pruebas de inteligencia.

La perspectiva del ciclo vital aporta una visión positiva de las capacidades intelectuales de las personas mayores. Esta visión positiva conlleva la creencia de que, en edades avanzadas, el aprendizaje es posible y por tanto la educación de las personas mayores debe orientarse al aprovechamiento de las capacidades y no a la compensación del déficit. Para lograrlo, los responsables de la educación deben partir de una consideración positiva de las capacidades de los mayores, acompañada de los recursos didácticos apropiados, lo que llevará a una mejora significativa del aprendizaje y a evitar bloqueos paralizantes (Flecha,1997).

Podemos así contemplar el aprendizaje como un proceso a lo largo de la vida, por lo que la participación en estudios formales para personas adultas mayores supone un estímulo para su crecimiento personal. La esperanza de vida después de la jubilación es cada vez mayor, por lo que estas personas suelen ser activas hasta edades muy avanzadas y por tanto se plantean ocupación del tiempo no sólo en actividades recreativas sino en formación y reciclaje. Para Willis (1985), un objetivo educacional es generar roles satisfactorios en la jubilación. En este caso la principal meta está en la satisfacción personal en vez de la económica. Las personas mayores muchas veces se inscriben en cursos y aulas Ilamadas de la Tercera Edad para que les ayuden a desarrollar metas para su tiempo de ocio tras la jubilación.

Cada vez con mayor frecuencia investigadores y profesionales desarrollan programas de enseñanza cognitiva y estrategias que están destinados a evitar o cambiar el sentido del déficit en el aprendizaje, la memoria o la resolución de problemas, por ejemplo, los talleres de memoria para personas ancianas sin ningún deterioro cognitivo ayudan a que estas personas puedan utilizar estrategias que les ayuden a mejorar su memoria. Los cursos de preparación a la jubilación también son un ejemplo de actividad de aprendizaje y con el avance de las nuevas tecnologías se han organizado cursos de formación en conocimientos informáticos para personas mayores. La formación en cuestiones relacionadas con el ordenador puede representar una área de potencial interés para las personas mayores y una vía para integrarse dentro de la corriente de nuevos conocimientos. En este caso formarse significa ser consciente de los cambios que experimenta la sociedad y, por tanto, implicarnos y participar de dichos cambios, apartando la imagen típica de la persona mayor desvinculada del resto de colectivos (Villar y Vilar, 2000). 
Con la formación, las personas mayores incrementan el bienestar y la confianza en sí mismas y se va perdiendo el estereotipo social de las personas ancianas apartadas de la formación y relegadas a la consideración más acorde con una visión deficitaria de la vejez que con la visión positiva que creemos debe potenciarse y que avalan las recientes teórias psicológicas.

\section{Referencias Bibliográficas}

ACHLEY, R. (1989) A continuity theory of normal aging. Gerontologist, 29, 183-190.

BALTES, P. B.: REESE, H. W.; NESSELROADE, J. R. (1981) Métodos de investigación en psicología evolutiva. Enfoque del Ciclo Vital. Madrid: Morata

BALTES, P. B. (1983) Psicología evolutiva del Ciclo Vital; Algunas observaciones convergentes sobre historia y teoria. En A. Marchesi, M. Carretero, J. Palacios (Ed). Psicología Evolutiva l; teorías y Métodos. Madrid: Alianza

BALTES, P. B.; BALTES, M. M. (1990) Psychological perspectives on successful aging: The model of selective optimitzation with compensation. En P.B. Baltes, M.M. Baltes (Eds) Successful aging. Perspectives from the behavioral sciences. Cambridge: Cambridge University Press.

BALTES, P. B. (1997) On the incomplete architecture of human ontogeny. American Psychologist, 52, (366-380)

BERGER, K. S.; THOMPSON, R. A. (2000) Psicología del desarrollo: Adultez y vejez. Madrid: Editorial médica panamericana.

BIRREN, J. A. (1961) A brief history of the psychology of Aging. Gerontologist, 1, 6771.

BRONFENBRENNER, U. (1986) La ecología del desarrollo humano. Barcelona: Paidós. (Edic. original inglesa 1979)

CASPI, A.; HERBENER, E. S.; OZER, D. J. (1992) Shared experiences and the similarities of personalities: A longitudinal study of married couples. Journal Personality and social Psychology, 62, 291-291.

CORRALIZA RODRÍGUEZ, J. A. (2000) Vejez y sociedad: dimensiones psicosociales. En R. Fernández Ballesteros (Ed.) Gerontologĺa social. Madrid: Pirámide.

FERNÁNDEZ BALLESTEROS, R. (1998) Calidad de vida en la vejez. Ponencias V Jornadas AMG. Barcelona.

FERNÁNDEZ BALLESTEROS, R. (2000) "La gerontología positiva" Revista Multidisciplinar de Gerontología. 10, 43-145

FLECHA, R. (1997) Compensación, Aceleración, inteligencia cultural. En J.García Carrasco. (Ed.) Educación de adultos. Barcelona: Ariel.

GRANJEL, L. S. (1991) Historia de la vejez. Gerontología, Gerocultura, Geriatría. Salamanca: Ediciones Universidad de Salamanca.

HORN, J. L.; CATTELL, R. B. (1966) Refinement and test of the theory of fluid and cristallized intelligence. Journal of Educational psychology. 57, 253-270 
HORN, J. L. (1982) Aging of fluid and cristallized intelligence. En F.I.M.Craik, S. Trehub (Eds.) Aging and cognitive processes. New York: Plenum Press.

HUYCK, M. H.; HOYER, W. F. (1982) Adult development and Aging. C. Belmont. Wadoworth.

INSERSO (1995) Cuidados en la vejez. El apoyo informal. Madrid: Inserso.

LEHR, U. (1988) Psicología de la senectud. Barcelona: Herder

LÓPEZ, F. (1998) Evolución de los vínculos de apego en las relaciones familiares. En M.J. Rodrigo, J.Palacios. (Eds). Familia y desarrollo humano. Madrid: Alianza.

MANGEN, D. I.; BENGSTON, V. L.; LANDRY, P. H. (1988). Measurement of intergenerational relations. Newbury Park, C.A.: Sage

PALACIOS, J. (1999) Introducción conceptual a la psicología evolutiva. En C. Triadó (Ed.) Psicología evolutiva. Vic: Eumo

RODRIGO, M. J.; PALACIOS, J. (1998) Familia y desarrollo humano. Madrid: Alianza.

RODRÍGUEZ DOMÍNGUEZ, S. (1989) La vejez historia y actualidad. Salamanca: Ediciones de la Universidad de Salamanca.

SCHAIE, K. W. (1989) The hazards of cognitive aging. The Gerontologist, 29, 484493.

SCHAIE, K. W. (1994) The course of adult intellectual development. American Psychologist, 49, 304-313.

SCHULTZ, R; HECKHAUSEN, J. (1996) A life Span model of successful aging. American psychologist, 51, 702-714.

TRIADÓ, C. (1997) Alternativas residenciales de las personas mayores. Anuario de Psicología, 73, 43-55.

TRIADÓ, C.; MARTÍNEZ, G. ; VILLAR, F. (2000) Psicología del desenvolupament: adolescència, maduresa $i$ senectut. Barcelona: Edicions Universitat de Barcelona

TRIADÓ, C.; VILLAR, F. (2000) El rol de abuelo: cómo perciben los abuelos a sus nietos. Revista española de Geriatría y Gerontología, 35, 30-36

VEGA, J. L. ; BUENO, B. (1995) Desarrollo adulto y envejecimiento. Madrid: Síntesis

VILLAR, F. (1998) Representación social del envejecimiento a lo largo del ciclo vital. Universidad de Barcelona. (Tesis doctoral no publicada)

VILLAR, F., VILÀR, J. (1999) Diseño y evaluación de un curso de informática y comunicación a su alcance, Revista Multidisciplinar de gerontología, 9,177-182.

VILLAR, F. (2000) ¿Tiene sentido la formación en la vejez? Las TIC como ejemplo paradigmático. Comunicación y Pedagogía (en prensa) 